\title{
Anabases
}

ANABASES Traditions et réceptions de l'Antiquité

22 | 2015

Varia

\section{À propos du voyage de Meillet en Arménie (1891,} 1903)

\section{Pierre Ragot}

\section{(2) OpenEdition}

1 Journals

Édition électronique

URL : http://journals.openedition.org/anabases/5501

DOI : 10.4000/anabases.5501

ISSN : 2256-9421

Éditeur

E.R.A.S.M.E.

\section{Édition imprimée}

Date de publication : 20 octobre 2015

Pagination : 233-242

ISSN : 1774-4296

\section{Référence électronique}

Pierre Ragot, « À propos du voyage de Meillet en Arménie (1891, 1903) », Anabases [En ligne], 22 |

2015, mis en ligne le 20 octobre 2018, consulté le 21 octobre 2019. URL : http://

journals.openedition.org/anabases/5501 ; DOI : 10.4000/anabases.5501 


\section{Archives de savants}

\section{À propos du voyage de Meillet en Arménie $(1891,1903)^{1}$}

\section{Pierre Ragot}

Élève de Louis Havet, Michel Bréal et Ferdinand de Saussure, Antoine Meillet (I866I936) peut être considéré comme le créateur de l'arménologie moderne ${ }^{2}$ et le fondateur ${ }^{3}$ de l'école française de linguistique et de grammaire comparée des langues indo-européennes ${ }^{4}$. Parmi ses élèves, il compte en effet presque tous ceux qui se sont consacrés à l'étude des langues dans la France de la première moitié du xx siècle ${ }^{5}$. Convaincu par ailleurs que le progrès scientifique repose aussi sur une bonne collaboration internatio-

1 Francis Gandon (éd.), Meillet en Arménie. Journaux et correspondance (I8gI, Igo3). Avec la collaboration d'Anne-Marguerite Fryba-Reber, Limoges, Lambert-Lucas, 2014, 290 p., ISBN 978-2-35935-o7i-5, 36€.

2 Sur ce point, voir G. Bolognesi, «Il contributo di Antoine Meillet agli studi di linguistica armena", in L'opera scientifica di Antoine Meillet (= osAm). Atti del Convegno della Società Italiana di Glottologia. Testi raccolti a cura di A. Q. Moreschini, Pisa, Giardini, I987, p. II9-I46 et à Ch. de Lamberterie, “Meillet et l'arménien », in Antoine Meillet et la linguistique de son temps, éd. S. Auroux, Histoire Épistémologie Langage Io/2 (1988), p. 2I7-234 et «La place de l'arménien dans la vie et l'œuvre d'Antoine Meillet », in Meillet aujourd'hui (= ma), éd. G.Bergounioux et Ch. de Lamberterie, Leuven-Paris, Peeters, 2006, p. I47-I89.

3 K. JABERG, "Hugo Schuchardt », Sprachwissenschaftliche Forschungen und Erlebnisse I (I937), p.3o3, voyait d'ailleurs en Meillet «le pater patriae de la linguistique en France» (der linguistische pater patriae Frankreichs).

4 Sur l'école française de linguistique, voir T. Bolleli: «La scuola linguistica sociologica francese », Studi e Saggi Linguistici I9 (I979), p. I-26.

5 Sur ce point, voir J. Vendryes, “Antoine Meillet», Bulletin de la Société de Linguistique (de Paris) 38/I (I937), p. 32-34. 
nale, son rayonnement et son influence à l'étranger furent considérables ${ }^{6}$. Meillet, qui maîtrisait de première main la quasi totalité des langues indo-européennes connues à son époque, a traité de toutes les grandes questions de phonétique et de morphologie indoeuropéenne en façonnant une méthode heuristique qui garde encore aujourd'hui toute sa valeur $^{7}$. Aussi son œuvre scientifique ${ }^{8}$ continue-t-elle à susciter un vif intérêt ${ }^{9}$.

Malgré l'influence et l'envergure de son œuvre et en dépit du portrait esquissé ici et là par certains de ses plus proches amis et collaborateurs ${ }^{10}$, la figure de l'homme et du savant dans son temps reste largement méconnue, même des spécialistes de l'histoire de la linguistique, car, si l'on fait abstraction d'une lecture publique faite quelques mois avant sa mort ${ }^{11}$

6 Comme en témoigne la liste des académies et sociétés savantes étrangères dont il était membre ainsi que celle de ses nombreux étudiants étrangers: cf. VENDrYEs, “Antoine Meillet », p. I2-I3 et 35-36.

7 C'est notamment le cas de son article, “De quelques difficultés de la théorie des gutturales indo-européennes ", Mémoires de la Société de Linguistique (de Paris) 8 (I89394), p.277-3o4, dans lequel il privilégie la reconstruction de deux ordres de dorsales (labio-vélaires et palato-vélaires) au lieu de trois (labio-vélaires, vélaires et palatales), et dont la "doctrine» fait désormais partie des acquis définitifs de la linguistique comparative indo-européenne: cf. p. ex. P. Ragot, Bulletin de la Société de Linguistique IOg/2 (20I4), p. I2I-I22.

8 Voir É. Benveniste, “Bibliographie des travaux d'Antoine Meillet», Bulletin de la Société de Linguistique 38/I (I937), p.43-68, liste reprise dans MA (n. I), p.3ı-338, puis corrigée et augmentée par P. Swiggers, ibid., p.339-354, qu'il faut compléter avec l'article de J. LoicQ, «Mémorial Antoine Meillet publié à l'occasion du centenaire de sa nomination au Collège de France (ıgo6-20o6)» Studia Indo-Europaea 3, 20o6, p. 5-ı69 et les “Compléments à la bibliographie d'Antoine Meillet », publiés ici même p. 242-246. P. Swiggers nous indique en outre (courriel du 27.Ir.20I4) que la liste intégrale des écrits de Meillet, paraîtra courant 2015 sous le titre Album Antoine Meillet: Bibliographie et études, éd. L. Isebaert, J.Loicq et P. Swiggers, Leuven-Paris, Peeters.

9 On aura un bon aperçu de l'œuvre de Meillet et des questions qu'elle suscite en se reportant à $\operatorname{OSAM}$ (n. I), à Histoire Épistémologie Langage Io/2 (I988), passim et à MA (n. I), p. I07-3og.

10 Les témoignages les plus importants sont, à nos yeux, ceux de Vendryes, “Antoine Meillet» (n. 4), p. I-42, de P.Boyer, “Antoine Meillet. I. L'homme et le savant», Revue des Études Slaves I6/3-4(I936), p. I9I-I98 et de L. Mariès, “Le sens qu'avait Antoine Meillet de l'arménien classique ", in Une cérémonie consacrée à la mémoire du Professeur Antoine Meillet, Paris, Publications de la Bibliothèque arménienne Nubar n ${ }^{\circ}$ I, I937, p. 28-49. On pourra également tirer profit de l'hommage d'A. MerLin, «Notice sur la vie et les travaux de M. Antoine Meillet », CRAI, I952, p. I-I4.

11 Il s'agit de la lettre de Meillet figurant in Célébration solennelle du quinzième centenaire de la traduction arménienne de la Bible au Grand Amphithéâtre de la Sorbonne, le dimanche 29 mars ig36. Lettres d'Antoine Meillet et de Charles Diehl. Discours de Mgr. Garékin Hovsepian, M.M. A.Tchobanian, N.Adontz, G.Millet et A.Gastoué, 
ainsi que des travaux d'Émile Benveniste ${ }^{12}$ et de Martiros Minassian (= M. M. $)^{13}$, les manuscrits et la correspondance de Meillet ont longtemps été inaccessibles aux érudits. Comme le rappelle à juste titre Anne-Marguerite Fryba-Reber ([= A.-M. F.-R.], p.I98-ı99), ce n'est qu'après la mort de la veuve de Meillet en 1975 que les archives de ce dernier furent déposées au Collège de France où elles furent classées et inventoriées par Simon Bouquet ${ }^{14}$ et Pierre Swiggers ${ }^{15}$. En 200I, elles furent toutes transférées à l'abbaye d'Ardenne (Calvados) avant d'être rapatriées au Collège de France en 20I3. Le dépouillement du Legs Meillet suscita rapidement l'intérêt de plusieurs savants et favorisa ainsi la publication de trois manuscrits

Publications de la Société des gens de lettres arméniens de Paris, Paris, Ernest Leroux, I938, p. I8-ı.

12 É. Benveniste, “Lettres de Ferdinand de Saussure à Antoine Meillet », Cahiers Ferdinand de Saussure 2I (I964), p.9I-I3o, correspondance complétée par R. JAковson, “La première lettre de Ferdinand de Saussure à Antoine Meillet sur les anagrammes ", L'Homme II/2 (I97I), p. I5-24 = Questions de poétique, Paris, Seuil, I973, p. I9o-20I et M. Minassian, «Sur la correspondance de Meillet avec Saussure relative aux anagrammes », Bulletin de la Société de Linguistique 7I/I (1976), p.35I-359 et « Saussure et les hypogrammes », Bulletin de la Société de Linguistique 72/I (1977), p. 34I-344.

13 Dans le cadre de sa traduction arménienne de l'intégralité des œuvres arménologiques de Meillet (cf. Hayagitakan usumnasirut'yunner [Études arménologiques], éd. M. Minassian, Erevan, I978), le prof. Minassian a eu, en ig63 et ig64, plusieurs contacts avec Mme Meillet, alors détentrice des archives de son mari. À l'occasion de ce travail, il a exhumé un compte-rendu inédit (cf. "Un inédit d'Antoine Meillet », Bulletin de la Société de Linguistique 70/I [1975], p.357-367), traduit en français un article qui n'était initialement paru qu'en arménien (cf. «Le locatif yamsean "dans le mois” en arménien classique », Bulletin de la Société de Linguistique 74/I [1979], p.333-334), et publié en arménien 7r des igo lettres et cartes postales que Meillet a adressées à des hommes de lettres arméniens et conservées au Musée d'Art et de Littérature d'Erevan (cf. “Avant-propos », in A. MeIllet, Lettres de Tiflis et d'Arménie. Du 29 avril au 3 août I8gI, présentées, annotées et publiées par M. Minassian, Vienne, Imprimerie Mékhitariste, I987, p. 6 n. II). Mme Meillet, pour sa part, souhaitait ardemment la publication des lettres adressées par Meillet à sa cousine Berthe. Aussi l'aida-t-elle à préparer l'édition susmentionnée en lui envoyant un exemplaire des copies de ces lettres dactylographié par le père Mariès (cf. ibid., p. I6-I7).

14 Voir S. Bouquet, «Les archives d'Antoine Meillet au Collège de France. Présentation et catalogue provisoire ", Archives et documents de la Société d'histoire et d'épistémologie des sciences du langage 8 (I988), p. II3-I40.

15 Voir P. Swiggers, «Les Archives Meillet au Collège de France: additions et corrections à l'inventaire », Bulletin de la Société de Linguistique 86/I (I99i), p.367-37o. 
inédits du grand linguiste ${ }^{16}$ ainsi que de plusieurs lettres de ou adressées à Meillet ${ }^{17}$. Le colloque qui lui fut consacré le 2I octobre 2000 à l'abbaye de Noirlac près de Châteaumeillant, où il possédait une demeure de famille et où il revenait régulièrement se ressourcer ${ }^{18}$, fut l'occasion de tirer deux de ses Journaux de l'oubli ${ }^{19}$.

L'ouvrage de Francis Gandon (= F. G.) que nous présentons ici s'inscrit dans le prolongement direct du dépouillement des archives arménologiques de Meillet initié par M.M. et poursuivi à l'occasion du colloque de Noirlac, puisqu'il permet au lecteur de découvrir le texte du Journal tenu par Meillet lors de son premier voyage en Arménie en i8gi et de relire à sa suite non seulement les cartes postales et lettres qu'il adressa alors à sa cousine Berthe $(=L B)$ et dont la diffusion initiale par les soins de M. M. était restée confidentielle ${ }^{20}$, mais également le texte du Journal rédigé à l'occasion de son second voyage dans le Caucase en I9o3, initialement publié par A.-M. F.-R. dans un volume destiné principalement à des spécialistes de linguistique historique et comparative ${ }^{21}$ et qui a pu échapper, de ce fait, à l'attention des historiens. L'ouvrage s'organise de la manière suivante: après la liste des abréviations des ouvrages et revues couramment cités (p.II-I2) et la “Note introductive» (p. I3-I4), on trouve la «Présentation générale» (p. I5-7i), le texte inédit du « Journal de voyage en Arménie (I89i)» (p. 73-ı8) puis les «Lettres à Berthe Esbaupin » (p. Io9-Ig6) suivies par la présentation remaniée de l'introduction au second Journal (p. 197-205) et le texte même du “Journal de voyage au Caucase (Igo3)» (p.206-22I), tous deux dus à A.-M. F.-R. Après les quatre «Annexes» (p.223-26I), l'ouvrage se termine par les «Références bibliographiques»

16 Voir A. Meillet, Pour un manuel de linguistique générale. Edizione di manoscritti inediti conservati al Collège de France, raccolti e pubblicati a cura di Fiorenza Granucci, Roma, Atti della Accademia nazionale dei Lincei, Anno CCCXII, I995, avec le compte rendu de Ch. de Lamberterie, Bulletin de la Société de linguistique 92/2 (1997), p. 20-24.

17 Voir p. ex. R. Amacker et S.Bouquet, "Correspondance Bally-Meillet (Igo6-rg32)», Cahiers Ferdinand de Saussure 43 (I989), p. 95-I20 et P. Swiggers, “La mort de Meillet», ${ }_{M A}\left(\mathrm{n}^{\circ}\right.$ 2), p.I37-I46. Cet article présente l'avantage de contenir (p. I/5-I/46) une liste commode des publications de l'auteur antérieurement dédiées à d'autres lettres de ou adressées à Meillet ainsi que la référence (p. I/4) de celles publiées avec la collaboration de P.Desmet. P. Swiggers nous fait par ailleurs savoir (courriel du 27/II/I4) que la parution de son livre La correspondance Antoine Meillet - Hugo Schuchardt est imminente. Voir Vendryes, “Antoine Meillet» ( $\left.n^{\circ} 5\right)$, p. 2-3 et Merlin, « Notice sur la vie et les travaux» (n. 9), p.3.

19 La première partie du volume $M A\left(n^{\circ} 2\right)$, qui constitue les actes de ce colloque, s'intitule “Les Journaux de Meillet» et occupe les p. a à io5. La présentation des deux Journaux est due à M. Fryba-Reber (p.3-ı), qui a également assuré seule l'édition et la transcription du Journal d'Arménie (p.87-105), tandis que celles du Journal personnel (p. 27-86) ont bénéficié de la collaboration de G. Bergounioux. Pour une vue d'ensemble du volume, on pourra se reporter au compte-rendu de Cl. Le Feuvre, Bulletin de la Société de Linguistique 102/2 (2007), p. 18-22.

20 Minassian, Lettres de Tiflis et d'Arménie (n. i3).

21 Les références exactes des actes du colloque de Noirlac sont indiquées supra, $\mathrm{n}^{\circ} 2$. 
(p.263-272), l'“Index des noms propres et notions » (p.273-288) et la “Table des matières » (p.289-29o).

Dans quelles circonstances Meillet a-t-il entrepris ses deux voyages (cf. p.I7-Ig et 27-33)? À partir de l'année universitaire I887-I888, Meillet s'initie à l'arménien classique en suivant les cours dispensés par Auguste Carrière à l'École des langues orientales. Bien que Carrière le considère comme le plus brillant de ses élèves, Meillet, qui veut devenir un arméniste confirmé, éprouve le besoin de parfaire sa formation à l'étranger. Durant l'hiver ı89o-I89i, il séjourne d'abord quelques semaines chez les pères Mékhitaristes de Vienne, un ordre catholique fondé par le moine Mékhitar de Sébaste au xvıı ${ }^{\mathrm{e}}$ siècle, mais il souhaite aussi voir l'Arménie, apprendre à parler arménien, étudier un dialecte arménien et se confronter à des manuscrits rédigés en arménien classique. Grâce à une maigre subvention, il s'embarque à Marseille le ı8 avril ı89ı pour aller jusqu'au port géorgien de Batoum en passant par Athènes (cf. p. 77-83) et Constantinople (cf. p.84-86), puis prend le train jusqu'à Tiflis, l'actuelle Tbilissi, où il arrive le 4mai (cf. p. I24). Jusqu'en I9ı7, la capitale de la Géorgie et des provinces caucasiennes de l'Empire russe fut peuplée d'une importante colonie arménienne. C'est dans ce foyer culturel arménien que Meillet apprend l'arménien moderne. Après une halte à Erevan, la capitale arménienne, il arrive à Etchmiadzine le ıojuin (cf. p. Io3 et I57). Centre religieux et siège du chef suprême de l'Église apostolique arménienne, le monastère d'Etchmiadzine est aussi renommé, à l'époque, pour sa bibliothèque de livres imprimés et notamment pour ses manuscrits arméniens. «Le voici adonné à la lecture et à la collation passionnante d'anciens manuscrits arméniens des évangiles, pendant des heures, dans le silence de la bibliothèque dont on lui a confié la clé [...] en quête de découvertes savantes relatives à l'orthographe ancienne et au texte arménien des Écritures ${ }^{22}$. „ Dès le 8 juillet (cf. p. I76), toutefois, il est installé à côté d'Etchmiadzine, à A(s) chtarak, pour y étudier le dialecte local. De tous les projets de publication caressés durant ce premier séjour en Arménie (cf. p. Ig), aucun ne verra le jour. Meillet se rend compte qu'il n'a ni le temps suffisant pour se familiariser avec le dialecte d'A(s)tarak ni la préparation nécessaire pour mener des investigations ardues sur les évangéliaires anciens ${ }^{23}$.

Tel ne se sera pas le cas douze ans plus tard. En rgo2, à la suite du décès de son maître Carrière, Meillet est nommé professeur d'arménien à l'École des langues orientales et rédige, en six semaines, à la demande de son maître viennois le père Dashian, sa fameuse Esquisse publiée l'année suivante chez les Mékhitaristes de Vienne ${ }^{24}$. Il demande alors une nouvelle mission en Arménie (cf. p. 200) et passe trois semaines (du 22juillet au ir août igo3)

22 Minassian, Lettre de Tiflis et d'Arménie (n. i3), p. I4.

23 La rédaction de ce passage est très redevable à Minassian, Lettres de Tiflis et d'Arménie (n. i3), p. iI-i4 et à de Ch. de Lamberterie, $m$ (n. 2), 2006, p. I53-i56.

24 Cf. Ch. de Lamberterie, ma (n. 2), 20o6, p. i6o. La référence exacte du livre mentionné est la suivante: A. Meillet, Esquisse d'une grammaire comparée de l'arménien classique,

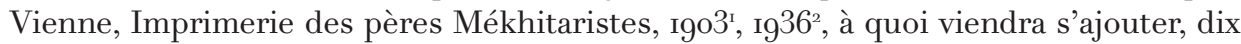
ans plus tard, le non moins remarquable Altarmenisches Elementarbuch, Heidelberg, Winter, I9I3, sur lequel voir également Lamberterie, ibid., p. i6i et I73-i75. 
à dépouiller les évangéliaires d'Etchmiadzine (cf. p.202). De ce second séjour arménien, il tirera trois grands articles toujours d'actualité 25 .

En dépit des nombreuses difficultés rencontrées pour (r)établir le texte original (cf. p. I6-I7, 73-74, IIо, 200 et 204), la lecture des trois documents présentés est agréable et très stimulante, car le savant rigoureux et austère cède le plus souvent le pas à l'homme cultivé (p. 8I, à propos des décorations dans l'architecture grecque: «Le mot de Fénelon: tourner les parties nécessaires en ornements naturels est une excellente définition de l'art grec»), curieux de tout mais clairvoyant (p.77: «J'ai un peu lu mon traité de psychologie: cette science-là n’a pas encore trouvé sa méthode»), voire au citoyen lucide (p. I45: «Le socialisme est [...] avant tout un acte de foi : le socialiste croit que l'homme a le pouvoir d'organiser la société de manière à la perfectionner et à effacer certains défauts [...]. L'impossibilité où l'on est de prévoir tous les résultats et de tout combiner n'est pas favorable à la théorie»), quand il ne s'efface pas totalement derrière l'homme pour se laisser aller parfois à des accès d'égoïsme (p. I72, à sa cousine Berthe: «Il est désolant que ta tante se remette ainsi: c'est pour retomber. Il vaudrait mieux en finir tout de suite [...]. Ne la prends pas chez toi [...]. Ce serait terrible et puis, si nous partons ensemble, qu'en ferais-tu? », cf. aussi p.I8o), et même à des angoisses bien légitimes, lorsque la succession de Saussure se trouve posée et que se joue alors son avenir professionnel (cf. p.i73 et I79-I8o), ou qu'un collègue, dont il a jugé sévèrement les ouvrages, cherche à le contacter (p. ıgı: «[...] je lui envoie l'adresse demandée avec une lettre la plus aimable possible. Je ne tiens pas à avoir un ennemi aussi important»), sans compter les moments où il fait part à sa cousine de son besoin d'affection (p. I84: “Une autre chose qui m’a été pénible, c'est de n'avoir personne auprès de moi pour se réjouir avec moi de mon succès » [sc. son élection à la succession de Saussure], cf. aussi p. 52$)$.

Sa capacité à condenser en des formules à la fois brèves et denses le fruit de sa réflexion, laquelle marquera de son empreinte indélébile son œuvre scientifique, affleure déjà dans cette quasi maxime qui rappelle la sagesse antique (p. I93): «Ne rien espérer de l'avenir et savoir supporter le présent sans colère et sans plainte, c'est presque l'idéal pour être heureux. » D'ailleurs, le savant n'est jamais loin et le linguiste fera son miel de ses réflexions pertinentes sur le bilinguisme (cf. p. Ioo), le difficile apprentissage d'une langue en immersion complète (cf. p. I29, I32, I37 et I5g) ou de cette réflexion, encore tout imprégnée de l'enseignement des néogrammairiens (p.2I8) : «Le savant est l'homme qui applique avec rigueur les formes de la pensée, qui fait rentrer les phénomènes dans les cadres précis de l'espace et du temps, qui cherche toujours la cause. »

L'intérêt de la présente édition tient aussi aux remarquables grilles de lecture dont elle est pourvue. Si l'intégration de la plupart des notes de M.M. à l'apparat critique propre au Journal de i8gi (cf. p. Iı3) permet de souligner la cohérence entre les propos contenus dans ce Journal et ceux figurant dans les $L B$, la substantielle introduction qui les précède offre au lecteur les rudiments de linguistique, de géographie, d'histoire, et de religion arméniennes dont il a besoin à la fois pour réinscrire les documents dans leur contexte mais aussi pour les dépasser et élargir sa réflexion. Ainsi, Meillet déplore-t-il à maintes reprises le peu d'enthousiasme des Arméniens pour leur langue et leur culture et dénonce même

25 Voir G. Bolognesi, $O S A M\left(\mathrm{n}^{\circ}\right.$ i), p. i 44 n. 93, et Ch. de Lamberterie, ibid., p. i6o n. 40. 
leur tropisme russe, qui n'a d'ailleurs d'égal que sa propre russophobie qui s'est encore accrue douze ans plus tard (cf. p. 2I5), parce que, faisant fi du contexte historico-politique, il «n'a pas vu qu'il ne s'agit pas tant d'une adhésion à un relatif Occident que d'un adieu symbolique à un joug oriental (perse et turc) dont l'Arménie a eu - et reste avoir - tant à souffrir. La dimension religieuse, sous-estimée par notre libre-penseur, est cardinale: quelque persécutrice que soit l'orthodoxie russe, c'est une orthodoxie chrétienne, partant non génocidaire» (p.5i ${ }^{26}$. Les trahisons dont l'Arménie a constamment été l'objet au cours de son histoire de la part de l'Occident chrétien ne s'expliqueraient-elles pas, en définitive, par son attachement à la doctrine du monophysisme que les Églises grecque et latine tenaient pour hérétique (cf. p.39-48) ${ }^{27}$ ? À la lecture de cette riche introduction, on apprend donc beaucoup.

Selon la précédente édition des $L B$, la cousine de Meillet s'appellerait Berthe Espau$\underline{\text { bin }}{ }^{28}$, mais la citation sur laquelle s'appuie M.M. est fautive. Dans le passage cité, Paul Boyer parle de «Mademoiselle Berthe Es $\underline{b}$ aupin ${ }^{29}$ ». On saura gré à F. G. d'avoir corrigé une erreur qui n'eût pas manqué de froisser Meillet, tant sa cousine, qui partage ses goûts pour la musique, la peinture et la langue russe, a joué un rôle important dans sa vie, comme le prouve sans conteste l'extrait d'une lettre de Saussure à Meillet consécutive au décès de Berthe, le ıा mars IgII (cf. p. III). D’ailleurs, plus son premier séjour arménien se prolonge, moins il parvient à cacher ses sentiments. On n'est donc pas surpris que Meillet ait également correspondu avec Berthe durant son séjour de Igo3 (cf. p.2I8). Une note indiquant si on possède ou non cette seconde correspondance aurait été la bienvenue.

La complémentarité entre le Journal de i8gi et les $L B$ aurait pu encore être renforcée avec quelques renvois internes supplémentaires. P. $77 l .29$ et p. $87 l l$. I-6, il conviendrait d'ajouter une note renvoyant p. I2I $l$. I6sqq., où il est aussi question du traité de psychologie que Meillet est en train de lire. Parallèlement, il faudrait préciser que l'extrait de la $L B$ citée p. 87 n. 2 se trouve p. I22. P. I9 n. 2, F. G. écrit: “Nous reparlerons de ce refus très général des lauréats de l'agrégation [...] d'enseigner en lycée [...] » : étant donné l'expression employée, un renvoi p.49, p. II2 n. 4, p. I8I et réciproquement est incontournable. P. 2II n. I, il convient de remplacer le renvoi à l'édition de M. M. par celui à la p. 163 de l'édition de F. G. et de répéter ce renvoi à l'aide d'une note supplémentaire p.212 $l$. 3. Dans la «Présentation générale» (p. I9), la liste des travaux que Meillet a tirés de son second séjour arménien n'est pas

26 Cet aveuglement de jeunesse contraste fortement avec son engagement ultérieur en faveur de la cause arménienne et le sens politique dont il sut faire preuve alors: sur ce point, voir Ch. de Lamberterie, $M A$ (n 2), 2006, p. I83-i85.

27 Cette passionnante analyse doit toutefois être nuancée car, au sein même de l'Église arménienne, de nombreux groupes ont adhéré au Concile de Chalcédoine : sur ce point, voir B.L. ZeKIYAN, “ Quelques réflexions préliminaires sur l'identité chrétienne de l'Arménie : l'universalité de la parole et son incarnation dans la vie de l'Ethnos ", Connaissance des Pères de l'Église 8I (200I), p. 2I-37, en part. p. 25.

28 Cf. Minassian, Hayagitakan Usumnasirut'yunner (n. I2), p. 8, suivi par Ch. de Lamberterie Histoire Épistémologie Langage, Io/2 (I988), p. 23ı n. 27 et, $M A$ (n. 2), 2006, p. 156.

29 Voir Boyer, “Antoine Meillet» (n. 9), p. I97 avec confirmation chez MerLin, «Notice sur la vie et les travaux» (n. 20), p.4. 
correcte. Il est en effet peu probable que les articles parus dans MSL I2, I9o3, p.429-43ı et MSL I3, I9o5, p.375 ait un quelconque rapport avec ce séjour et la liste fournie p.58 est partiellement incorrecte et incomplète: $l$. Io, il faut supprimer le renvoi à «1904» et à « $1905 \mathrm{~b}$ » $=M S L$ I3, I905, p.375), ajouter celui à “I903e» et conserver celui à «I905c» et corriger en conséquence la p. I ${ }^{30}$. P. I67-ı68, un renvoi externe à l'article de H. Bat-Zeev Shyldkrot, “Antoine Meillet et les langues romanes ", $H E L$ Io/2, I988, p. 2I2-2I3, s'impose, puisque Meillet expose ici la conception de la diction de la poésie française qu'il défendra à plusieurs reprises dans ses comptes-rendus d'ouvrages portant sur la stylistique et la linguistique françaises.

Si certains passages mal rédigés peuvent susciter la perplexité (cf. p. 66 ll. 23-27, p.68 ll. I9-27 et p. I19 $l l$. I8-22), le lecteur attentif corrigera aisément les quelques coquilles qui sont à déplorer. Signalons toutefois que la création en i828 d'une province arménienne de Russie puis sa fusion en I840 dans celle d'Imérétie n'est pas l'œuvre de Nicolas II (sic! p.34 ll. 3i et 37), tsar de I894 à I9I7, mais celle de son arrière-grand-père Nicolas I ${ }^{\text {er }}$, tsar de I825 à I855 et précisons que la plaquette à laquelle nous faisons allusion n. Io et dont la couverture est reproduite p.23, n'est pas citée correctement dans la bibliographie p. 269. On corrigera également une partie de la n. I p.23: la célébration du quinzième centenaire de la traduction arménienne de la Bible a lieu le 29mars ig36, tandis que Meillet est décédé le 2i septembre I936. Ce n'est donc pas la lettre de Meillet qui est "posthume» mais sa publication en Ig38. Une expression comme “publiée à titre posthume» aurait levé toute ambiguïté.

On regrettera également que F. G. se laisse parfois aller à créer des néologismes maladroits, tels "graphié» (p.20 l. 26) au lieu de «mis par écrit» et «insusceptible de» (p.66 l. I7 et p. II3 n. I $l$. 8) au lieu de “non susceptible de», et ait décidé de remplacer les noms "différence» et “écart» par le vieux substantif du xıi ${ }^{e}$ siècle “discrépance» (p.27l. I8 et p.25I $l$. II) qui, depuis qu'il a été acclimaté outre Manche sous la forme discrepancy, ne peut manquer de passer pour un anglicisme superflu. Parfois, le recours à certains termes techniques obscurcit inutilement son propos. Tel est le cas du nom «spéciation» (p.7o ll. I3 et I4), qui, pour reprendre la définition de Cl. Hagège, désigne «les traits particuliers acquis au cours de l'évolution par des espèces vivant en milieu isolé31 ». Dans le contexte, une expression comme “l'émergence des caractéristiques propres à une langue» ne serait-elle pas plus claire? Lorsque (p. 7i n. I) l'auteur se demande avec raison si l'on peut véritablement distinguer, dans une langue, ce qui s'expliquerait par les seules structures linguistiques de ce qui relèverait aussi du fait social (et culturel) cher à Meillet, est-il indispensable de parler, dans le premier cas, de «basilectal» et, dans le second, d'“ acrolectal»? D'autre part, à notre connaissance, l'élève de Meillet, Hratchia Adjarian (I876-I953), a rédigé son Dictionnaire étymologique de l'arménien cité à deux reprises (p. 2Io n. I et p.263) en arménien moderne oriental. Il vaudrait donc mieux écrire Hayerēn armațakan ba iaran plutôt que de recourir à l'arménien moderne occidental Hayerēn armadakan pa-iaran.

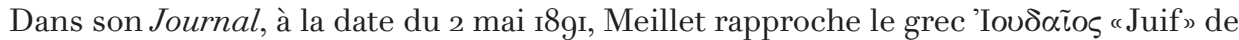
son correspondant arménien et rend compte de ce dernier en postulant la chute de $u$ et le passage de $d$ à $r$ (cf. p.88). F. G. veut voir dans cette fulgurante intuition matinale les

30 Sur la question des publications que Meillet a tirées de son second séjourà Etchmiadzine en I9o3, voir aussi la réf. supra, n. 26.

31 Voir Cl. Hagège, Contre la pensée unique, Paris, Odile Jacob, 20I2, p. I35. 
prémisses de ce qui deviendra la «loi de Meillet». Pour étayer son propos, il commence par reproduire (“Annexe I», p. 223-247) l'excellent article que Ch. de Lamberterie a consacré à cette loi $^{32}$ et en déduit (“Annexe 2», p.25I) que l'arménien Ereay (sic!) s'explique lui aussi par la «loi de Meillet». Ce raisonnement ne tient pas, car l'étude de Ch. de Lamberterie montre clairement que la «loi de Meillet», qui, rappelons-le, postule, en se fondant sur le cardinal “deux», qu'un groupe initial indo-européen * $d w$ - aboutit à erk- en arménien, ne touche que les mots arméniens hérités du vieux fonds indo-européen, en aucun cas les emprunts. De plus, la forme que Meillet transcrit dans son Journal est erronée. Ainsi que F. G. l'indique en note (cf. p.88 n. I et 2), il faut lire Hreay. Comme l'a expliqué Meillet luimême ${ }^{33}$, le passage de - $d$ - à - $r$ - trahit en réalité, dans ce terme comme dans d'autres, un

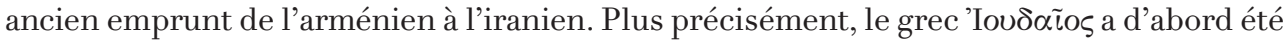
emprunté par le syriaque sous la forme $\bar{\imath} h \bar{u} \partial \bar{a} y \bar{a}$ avant d'être transmis à l'arménien par un intermédiaire parthe dont nous n'avons pas la trace, si ce n'est précisément à travers le passage de $-\delta-$ à $-r_{-}{ }^{34}$. Ce traitement phonétique, qui est exactement parallèle à celui qu'a subi la consonne ${ }^{*} d$ - dans le groupe indo-européen $* d \omega$ - au cours de son évolution en arménien (cf. p.240-24I), est, en définitive, le seul point commun que l'on puisse déceler avec la «loi de Meillet».

Les quelques critiques qui viennent d'être émises ne remettent évidemment pas en cause la qualité de l'ensemble de l'ouvrage. Elles attestent avant tout de l'intérêt que nous avons eu à le lire. Pour remettre dans leur contexte les documents publiés, F. G. a été contraint de solliciter l'aide de collègues d'autres spécialités (notamment les deux arménologues Anaïd Donabedian et Agnès Ouzounian) et de s'aventurer dans des domaines qui ne sont pas les siens. La somme de travail qu'il s'est imposée est considérable, mais elle n'a pas été vaine: le résultat est là, éclatant. Nul doute donc que ce livre, à la fois facile à se procurer et richement documenté, fera date dans les études meilletiennes. Souhaitons que l'auteur puisse publier bientôt le reste de la correspondance d'Arménie (lettres de Meillet à son père et à ses collègues restés en France dont celles adressées à son ami Paul Boyer et A. Meillet, Études de linguistique et de philologie arméniennes, vol. II, Louvain, Imprimerie orientaliste, I977, p.26r.

34 Détails complémentaires chez G. Bolognesi, Le fonti dialettali degli imprestiti iranici in armeno, Milano, Società Editrice Vita e Pensiero, I96o, p. 66-67 et R. Sснмітт, Grammatik des Klassisch-Armenischen mit sprachvergleichenden Erläuterungen, Innsbruck, Innsbrucker Beiträge zur Sprachwissenschaft, I98I, p. I7I-I72. En définitive, l'emprunt parthe prend, en proto-arménien, la forme *Hureay, laquelle évolue régulièrement en Hreay après la chute de l'-u- en syllabe prétonique: cf. Meillet, Esquisse d'une grammaire (n. 24), I936², p. 20. Pour une vue d'ensemble très commode du passage de - $\partial$ - à - $r$ - dans les emprunts arméniens au parthe, voir aussi G. Bolognesi, OSAM (n. I) p. I34 et p. I38-I40. Dernier état de la question chez M. Morani, “Alcune riflessioni sui prestiti siriaci in armeno ", in He Bitaney Lagge. Studies on Language and African Linguistics in Honour of Marcello Lamberti, a cura di L. Busetto, R. Sottile, L. Tonelli e M. Tosco, Milan, Quasar s.r.l., 20II, p. I3o. 
à son maître Auguste Carrière) et que son travail suscite d'autres vocations pour explorer les fonds d'archives des savants français et étrangers susceptibles de contenir des lettres de Meillet ${ }^{35}$. C'est à ce prix que l'on pourra rendre à Meillet toute la place qu'il mérite dans l'historiographie de la première moitié du $\mathrm{xx}^{\mathrm{e}}$ siècle.

Pierre Ragot pierrehenri.ragot@wanadoo.fr

\section{Compléments au Mémorial Antoine Meillet}

\section{Jean LoicQ}

Les notes qui suivent visent à compléter la documentation réunie dans le Mémorial Antoine Meillet publié à l'occasion du centenaire de son entrée au Collège de France (Igo6), dans Studia Indoeuropaea. Revue de mythologie et de linguistique comparée, t. III (2006), et à part (Bucarest). La bibliographie propre d'A. Meillet qui figure dans ce Mémorial se voulait elle-même un complément de celle, déjà très imposante, qu'au lendemain de sa mort son successeur Ém. Benveniste avait hâtivement dressée en appendice au long article biographique de $\mathrm{J}$. Vendryes ${ }^{36}$, et qui s'est révélée très incomplète.

Sans doute, en effet, l'œuvre de Meillet (r866-ıg36) est essentiellement celle du linguiste qui a dominé sa discipline durant le premier tiers du $\mathrm{xx}^{\mathrm{e}}$ siècle, explorant toutes les branches de la famille indo-européenne, œuvre dont l'essentiel ne pouvait échapper à la vigilance de Benveniste. Mais la curiosité de Meillet et son action n'en ont pas moins porté sur des aspects des sciences de l'homme et de l'actualité où la science du linguiste et l'impartialité du savant lui conféraient une autorité incontestée.

On a pu voir ici-même par la recension de P. Ragot quel intérêt il portait à la culture arménienne: non seulement à sa langue, qu'il a un temps enseignée à l'École des Langues Orientales (auj. l'INALCO), mais aussi à sa littérature et à son art médiéval, avec une prédilection pour son architecture religieuse, dont il appréciait la pureté et la netteté de lignes. Il importe de rappeler aujourd'hui, en cette année d'un centenaire douloureux, que dès la fin de la Première Guerre, et surtout à la veille de la Conférence de Lausanne (I923), Meillet a mis avec persévérance son autorité au service de la cause arménienne. Avec son successeur à l'École des Langues, Frédéric Macler, il devait fonder dès I92o la Revue des études

35 Ce type de recherche reste toutefois aléatoire. Ainsi, bien qu'Antoine Meillet ait entretenu une correspondance suivie avec son collègue et ami Joseph Vendryes, le Fonds Vendryes ne contient aucune lettre de Meillet, d'après l'enquête préliminaire menée par P.-Y. Testenoire, “Les manuscrits de Joseph Vendryes : premier état des lieux", in Archives et manuscrits de linguistes, éd. V. Chepiga et E. Sofia, Louvain-laNeuve, Academia-L'Harmattan s.a., 2014, p. 96.. 